Recepción: 11 / 05 / 2018

Aceptación: 10 / 07 / 2018

Publicación: 05 / 10 / 2018

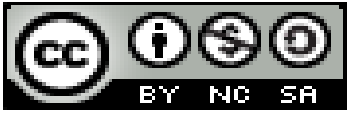

Ciencias de la educación Artículo de investigación

\title{
Herramienta multimedia educaplay como recurso didáctico en el proceso enseñanza- aprendizaje de química y física general
}

Educaplay multimedia tool as a teaching resource in the teaching process chemistry and general physics learning

Ferramenta educativa multimídia como recurso de ensino no processo de ensino - química e aprendizado em física geral

Monserrat Orrego-Riofrío ${ }^{\text {I }}$ morrego@unach.edu.ec

Carlos J. Aimacaña-Pinduisaca II caimacania@unach.edu.ec

Correspondencia: morrego@unach.edu.ec

\footnotetext{
${ }^{\text {I }}$ Magister en Docencia Universitaria e Investigación Educativa, Doctora en Química, Docente de la Universidad Nacional de Chimborazo, Riobamba, Ecuador.

II Magister en Gerencia de Proyectos Educativos y Sociales, Licenciado en Ciencias de la Educación Profesor de Enseñanza Media en la Especialización de Ciencias Exactas, Docente de la Universidad Nacional de Chimborazo, Riobamba, Ecuador.
} 


\section{Resumen}

Las tecnologías de la información y comunicación se encuentran todas las áreas del quehacer humano, sin embargo parece haber quedado fuera de las aulas, se sigue ensañando y aprendiendo de forma tradicional, La Universidad Nacional de Chimborazo está dispuesta cambiar ese paradigma y está consciente de que hoy más que nunca el uso de las TICS es una herramienta indispensable en el proceso docente educativo, el objetivo del presente trabajo es utilizar la herramienta multimedia (educaplay) como estrategia para el aprendizaje de química y física general, el diseño de la investigación fue no experimental, se trabajó con los 22 estudiantes del segundo semestre de la carrera de Biología, Química y Laboratorio con los que se trabajó intensamente con la elaboración y aplicación de actividades se utilizó la encuesta y se aplicó pruebas objetivas para medir el aprendizaje, se empleó la prueba t de students para muestras dependientes, para demostrar la relación entre el uso de educaplay y el logro de aprendizajes, finalmente de determinó que la herramienta multimedia educaplay impulsa el aprendizaje de química y física general.

Palabras clave: educaplay; recursos didácticos; aprendizaje.

\section{Abstract}

Information and communication technologies are found in all areas of human activity, however it seems to have been left out of the classroom, is still taught and taught traditionally, The National University of Chimborazo is willing to change that paradigm and is aware that Today more than ever the use of ICT is an indispensable tool in the educational process, the objective of this work is to use the multimedia tool (educaplay) as a strategy for the learning of chemistry and general physics, the design of the research was not experimental, we worked with the 22 students of the second semester of the Biology, Chemistry and Laboratory career the survey was used and objective tests were applied to measure the learning, the student's $t$ test was used for dependent samples, to demonstrate the relationship between the use of educaplay and the achievement of learning, finally determined that the tool multimedia educaplay promotes the learning of chemistry and general physics.

Key words: multimedia tool; strategy; learning. 


\section{Resumo}

A tecnologia da informação e comunicação são todas as áreas da atividade humana, no entanto, parece ter sido fora da sala de aula, continua ensañando e aprender forma tradicional, a Universidade Nacional de Chimborazo está disposto a mudar esse paradigma e está ciente de que hoje mais do que nunca a utilização das TIC é uma ferramenta indispensável no processo educacional, o objetivo deste trabalho é a utilização de ferramentas multimédia (Educaplay) como uma estratégia para a aprendizagem de química e física geral, o desenho da pesquisa não foi experimental, trabalhamos com os 22 alunos do segundo semestre da carreira de Biologia, Química e Laboratório, com quem trabalhamos intensamente com a preparação e aplicação das atividades, utilizamos a pesquisa e aplicamos testes objetivos para medir a aprendizagem, teste $\mathrm{t}$ de alunos para amostras dependentes, para demonstrar a relação entre o uso de educaplay e a realização de aprendizagem, finalmente determinou que a ferramenta educativa multimídia promove a aprendizagem de química e física geral.

Palavras chave: educaplay; recursos de ensino; aprendizagem.

\section{Introducción}

En la actualidad se habla de la revolución tecnológica en todos los campos, esencialmente en la educación, sin embargo en muchas aulas se sigue trabajando como hace un siglo atrás, utilizando los mismos recursos didácticos que en su mayoría son el lápiz, el papel, la pizarra, sin desmerecer su importancia dentro del aula, sin embargo son los principales causantes de la falta de dinamización en el aprendizaje lo que se refleja en el estudiante con la falta de interés a las clases impartidas, bajas notas, desmotivación, temor a la materia.

Hoy más que nunca el reto es cambiar esa realidad y es así que en el Ecuador ha aumentado el acceso a internet tanto en los hogares como en las instituciones educativas gracias a políticas públicas; por lo cual es coherente pensar y actuar a la par de la tecnología ofrecida, cambiando la utilización del clásico cuaderno u hojas de deberes por una computadora o lo más utilizado actualmente un Smartphone, que será el medio por el cual tanto, docentes y estudiantes interactúen. de igual forma se aumentado notablemente los procesos de capacitación que se dan 
en todos los niveles en relación a la inclusión de las TIC en el proceso docente educativo, no solo en el aula sino en los trabajos autónomos que los estudiantes realizan.

En la Facultad de Ciencias de la Educación Humanas y Tecnologías de la Universidad Nacional de Chimborazo se conoce la importancia de implementar las TIC en las actividades docentes, sin embargo esta inclusión aún no se consigue de forma eficiente, es así como en la población de segundo semestre de la Carrera de Biología Química y Laboratorio en la cual se basa la investigación se realizó una encuesta en la que se refleja un problema: el desconocimiento de los estudiantes sobre la herramienta multimedia Educaplay, a partir de esto surge la investigación como respuesta a dicho problema. Puesto que en el aula muchas veces se llega a una monotonía de actividades en las cuales siempre están inmersas el papel y el lápiz, sin usar las herramientas tecnológicas que favorecen un aprendizaje más activo y dinamizador.

El presente trabajo de investigación se basa en el constructivismo y en el aprendizaje significativo. Según Ausubel, se requiere dos condiciones esenciales para el aprendizaje: disposición del sujeto a aprender significativamente y material de aprendizaje potencialmente significativo.

La investigación que se realizó surge como respuesta al problema detectado y es una alternativa en la cual el uso de una computadora conectada a la web sea el medio para que ocurra el aprendizaje, pues brinda al estudiante diferentes fuentes: contenido científico, animaciones, imágenes, audio, video, simuladores, mapas mentales, etc. A partir de los cuales el estudiante toma un rol protagónico en cuál es el quien clasifica el material que va a utilizar, esto conlleva a que realice procesos mentales superiores y sea el quien construya sus propios aprendizajes, con la guía del docente, lo cual propicia el logro de aprendizajes que se ve reflejado en el rendimiento académico.

Al revisar trabajos similares realizados por otros autores como Hernández y Rodríguez (2014) donde indica que se facilita la comprensión de conceptos "cuando se emplean imágenes en movimiento (videoclips y/o películas) ya que estas describen fenómenos dinámicos y además se requiere menos tiempo para reflexionar"(p.39) se determinó que el actividades como videoquiz desarrolladas con la aplicación educaplay será favorable para mejorar el aprendizaje, y sobre todo 
permite lograr un cambio de actitud temerosa e indiferente que muchos estudiantes tienen hacia la química, y sus actividades favorece un aprendizaje cooperativo (Sepulveda, 2014, p. 106)

Con estas premisas, se va intentar responder ¿Cómo herramienta multimedia EDUCAPLAY como recurso didáctico propicia el aprendizaje de química y física general?

El objetivo del presente trabajo es determinar si el empleo la herramienta multimedia EDUCAPLAY como recurso didáctico mejora el rendimiento académico de las asignaturas de Química y física General en los estudiantes de segundo semestre de la carrera de Biología, Química y Laboratorio.

La hipótesis planteada fue: No hay diferencias en el rendimiento académico entre la medición de inicio y la medición hecha al finalizar el taller utilizando la herramienta educaplay como recurso didáctico.

Por tanto, los resultados de esta investigación pretenden fortalecer el uso de herramientas multimedia como el educaplay para la enseñanza de ciencias experimentales como la química y la física, que, en la Universidad Nacional de Chimborazo, Facultad de Ciencias de la Educación, Humanas y tecnologías se lo está haciendo de forma tradicional.

En la actualidad los docentes están cambiando su rol de proveedor debido a que el conocimiento esta online y ya no necesita quien lo suministre, y lo que hace falta es una figura quien refuerce conocimientos y guían la enseñanza, es por ese motivo que debemos acoplarnos a las necesidades individuales de los "nativos digitales" los cuales tienen a su disposición toda la información y esta hace que los estudiantes obtengan un poder, el cual con la guía adecuada el mundo puede tener un cambio positivo.

Utilizar las Tecnologías de la información y comunicación resulta vital en el siglo XXI, ya que las mismas ofrecen la posibilidad de recrear, y solucionar problemas del entorno, facilitando las actividades cotidianas que realiza el ser humano, y más aún en el sistema educativo que día a día cambiando, hace necesario el uso de recursos didácticos que sean atrayentes y motivantes para el educando si se considera lo expuesto por Riveros y Mendoza (2005) el uso de las TICs “ Permite que el alumno controle su ritmo de aprendizaje. El tiempo destinado a procesar, registrar, 
analizar, aplicar y evaluar un determinado material de aprendizaje puede ser regulado por el propio alumno. El contenido puede ser dosificado y secuenciado de acuerdo con sus necesidades y ritmo de aprendizaje (p.335)

Las ventajas que ofrecen las TIC para lograr una mejor comprensión de los contenidos, leyes y principios que se estudian dentro de la Química, son la posibilidad de utilizar diferentes formas de aprendizaje; la resolución de problemas ya que se facilita su representación mental tanto del problema como de la solución. (Gómez, 2012, p. 27)

La Química y Física corresponden a un ámbito importante del conocimiento científico; está formada por un cuerpo organizado, coherente e integrado de conocimientos. El proceso de aprendizaje de estas ciencias es particularmente importante en el nivel superior pues obedece a la necesidad de establecer un eslabón entre la formación científica y las exigencias del aprendizaje interdisciplinario.

En química y física se habla del aprendizaje constructivo y significativo debido a que permite el logro de objetivos y principalmente, el desarrollo de competencias, creando las condiciones necesarias para que los estudiantes, sean los protagonistas de su aprendizaje mediante el desarrollo de actividades tanto individuales como colaborativas; así como el que sean capaces de controlar sus propios procesos.

Las nuevas tecnologías entre ellas las herramientas multimedia son un recurso didáctico que nos permite aprovechar diversas posibilidades en distintas áreas y niveles educativos, para mejorar y dinamizar nuestra labor docente ayudando al estudiante a desarrollar destrezas y llevarlo a procesos mentales superiores.

Una de las ventajas que tiene el uso de herramientas multimedia con tecnología de punta y en línea es decir que utilicen internet, es la posibilidad de superar barreras tales como el tiempo y el espacio, permitiendo a los profesores y a alumnos una interacción en tiempo real para el intercambio de experiencias, consultas y construcción de conocimientos. Como lo indica las TIC en la universidad pública y privada, permite a los estudiantes fortalecer fe la aprehensión de conocimiento y el enriquecimiento cognitivo. (Riascos \& Quintero, 2009, p. 156) 
En la actualidad es muy común escuchar hablar sobre la tecnología en la educación, sin fijarse que estos medios tecnológicos han estado presentes desde hace mucho tiempo atrás, hablar de herramientas multimedia es referirse a objetos o sistemas físicos que permitan la emisión de la información, así tenemos por ejemplo la televisión, la radio, videos, etc. Todas estas herramientas han cumplido un gran valor en la educación así que en la actualidad se presentan programas de televisión y radio enfocados a la educación es el caso de "EDUCA" el cual se transmite de manera frecuente en nuestros medios de comunicación.

La aparición de las nuevas tecnologías ha abierto una nueva etapa metodológica en la educación, permitiendo que docentes y estudiantes utilicen muchas herramientas tecnológicas que antes no lo hacían en clase, lo cual favorece las oportunidades para desarrollar la investigación y realizar actividades colaborativas, lo cual favorece el desarrollo de competencias como la digital, tratamiento de la información y de aprender a aprender (Domingo y Pere, 2011, p.170), conocerlas y utilizarlas es hoy un factor clave en las aulas universitarias. Permite agregar a la educación un plus motivación, divertido y novedosos, el cual permite la dinamización de la educación gracias a sus características y a su amplio campo de acción pues se puede aplicar a diversas asignaturas, todo depende de la creatividad del docente o estudiante.

Educaplay, es una plataforma que permite al docente y estudiante diseñar un conjunto de actividades educativas online. (Pérez, 2014, p.24), cuenta al momento con 17 tipos de actividades didácticas y lúdicas a las cuales tienen un video tutorial el en el cual se describe paso a paso la forma de ejecutarlas, todos los usuarios tienen accesos a ellas, a continuación, se describe brevemente algunas actividades: Relacionar Mosaico, por medio de ésta actividad se puede relacionar parejas de elementos. Videoquiz, permite al alumno observar un video y durante su desarrollo contestar preguntas relacionadas con el mismo, o de aplicación de lo observado, lo cual favorece la retención y asociación de los conocimientos previos con los nuevos expuestos en el video, además, se puede trabajar con los estudiantes actividades lúdicas como sopa de letras, crucigramas, relacionar columnas, sopa de letras, videoquiz, test, mosaico, test, ruletas de palabras, etc. 


\section{Metodología}

La presente investigación fue no experimental debido a que no posee control sobre las variables, es decir no cambian intencionalmente, pues solo se observó los fenómenos tal y como son en su contexto natural, para luego analizarlos.

El tipo de investigación propuesta fue de Campo y Bibliográfica; de Campo debido a que la acción del investigador estuvo en contacto directo con las personas sobre las cuales se realizó el estudio en cuestión. Bibliográfica ya que consistió en la búsqueda, recopilación, organización, valorización, crítica e información de datos bibliográficos y proporciono el conocimiento de las investigaciones ya existentes a cerca del tema que se va a investigar.

El nivel de la investigación fue correlacional; ya que se determinó la relación entre el uso de la herramienta educaplay con el aprendizaje de química general.

El método que se aplicó en la investigación fue el método inductivo-deductivo se partió en la observación y la experimentación de sucesos que ocurrieron dentro del aula de clase, se elaboró y aplicó un conjunto de actividades usando educaplay tales como crucigramas, relacionar columnas, sopa de letras, videoquiz, test, mosaico, test, ruletas de palabras tanto para fortalecer el aprendizaje de los contenidos de las asignaturas de física y química, se evaluó los aprendizajes usando pruebas objetivas, antes durante y después de la aplicación, se promedió los resultados de dichas evaluaciones y se aplicó la prueba t para comparación de medias para muestras dependientes, con la finalidad de comprobar la hipótesis.

Se aplicó la técnica de observación con la finalidad de analizar los resultados de aprendizaje logrados por los estudiantes en las asignaturas de química y Física general, y comparar el uso de educaplay como recurso didáctico con la metodología tradicional.

Población objeto de estudio, estudiantes de Química y Física General del segundo semestre de la carrera de Biología, Química y Laboratorio.

La muestra que participó en la investigación estuvo compuesta por 22 estudiantes de las asignaturas de Química y Física General del segundo semestre de la carrera de Biología, Química 
y Laboratorio de la Facultad de Ciencias de la Educación Humanas y Tecnologías de la Universidad Nacional de Chimborazo. Se trabajó con toda la población ya que esta es pequeña, así los resultados de la investigación serán relevantes.

\section{Resultados y Discusión}

Con los resultados obtenidos de las evaluaciones de aprendizaje realizadas al inicio, y con las obtenidos durante y al final de la aplicación de las actividades con Educaplay, a los estudiantes de segundo semestre de la carrera de Biología, Química y Laboratorio, se procedió a determinar si las medias aritméticas de rendimiento académico son iguales.

Ho: No hay diferencias en el rendimiento académico entre la medición de inicio y la medición hecha al finalizar el taller utilizando la herramienta educaplay como recurso didáctico

Ha: hay diferencias en el rendimiento académico entre la medición de inicio y la medición hecha al finalizar el taller utilizando la herramienta educaplay como recurso didáctico.

\section{Prueba estadística empleada: Prueba t para muestras relacionadas}

Regla de decisión: Si $\mathrm{p} \leq 0.05$ se rechaza Ho, se acepta Ha

El nivel de significancia es 0.05 (que se establece en ciencias sociales y en psicología normalmente)

Se utiliza el programa informático estadístico SPSS se realiza la comparación de medias para muestras pareadas y los resultados se muestran en la tabla 1. 


\section{Tabla 1}

Resultados de la aplicación de la prueba t para muestras dependientes

\begin{tabular}{|l|r|r|r|c|}
\hline & Estadisticas de muestras emparejadas \\
\hline & Media & \multicolumn{1}{c|}{$\mathrm{N}$} & \multicolumn{1}{|c|}{$\begin{array}{c}\text { Desviación } \\
\text { estándar }\end{array}$} & $\begin{array}{c}\text { Media de } \\
\text { error } \\
\text { estándar }\end{array}$ \\
\hline Par 1 & $\begin{array}{l}\text { promedios antes de la } \\
\text { aplicación }\end{array}$ \\
$\begin{array}{l}\text { Promedios después de } \\
\text { la aplicación }\end{array}$ & 5.1818 & 22 & 2.28111 & .48633 \\
\hline
\end{tabular}

Correlaciones de muestras emparejadas
\begin{tabular}{|ll|r|r|r|}
\hline & \multicolumn{1}{|c|}{ N } & Correlación & Sig. \\
\hline Par1 & $\begin{array}{l}\text { promedios antes de ia } \\
\text { aplicación \& Promedios } \\
\text { después de la aplicación }\end{array}$ & 22 & .849 & .000 \\
\hline
\end{tabular}

\begin{tabular}{|c|c|c|c|c|c|c|c|c|c|}
\hline \multicolumn{10}{|c|}{ Prueba de muestras emparejadas } \\
\hline & & \multicolumn{5}{|c|}{ Diferencias emparejadas } & \multirow[b]{3}{*}{$\mathrm{t}$} & \multirow[b]{3}{*}{ أو } & \multirow[b]{3}{*}{ Sig. (bilateral) } \\
\hline & & \multirow[b]{2}{*}{ Media } & \multirow{2}{*}{$\begin{array}{c}\text { Desviación } \\
\text { estándar }\end{array}$} & \multirow{2}{*}{$\begin{array}{l}\text { Media de } \\
\text { ertor } \\
\text { estándar }\end{array}$} & \multicolumn{2}{|c|}{$\begin{array}{l}\text { 95\% de intervalo de confianza } \\
\text { de la diferencia }\end{array}$} & & & \\
\hline & & & & & Inferior & Superior & & & \\
\hline Par 1 & $\begin{array}{l}\text { promedios antes de la } \\
\text { aplicación-Promedios } \\
\text { después de la aplicación }\end{array}$ & -2.50000 & 1.26303 & .26928 & -3.05999 & -1.94001 & -9.284 & 21 & .000 \\
\hline
\end{tabular}

Fuente: Resultados de las evaluaciones de los estudiantes.

Se observa un valor de $\mathrm{t}$ de $-9.284, \mathrm{gl}=21$ grados de libertad y $\mathrm{p}=0.000$, menor que 0.05 por lo que se concluye que el rendimiento académico de los alumnos es diferente con el uso de la herramienta multimedia educaplay. En cuanto a la efectividad del uso de educaplay hay un incremento en la media de rendimiento de 5.18/10 antes del uso de educaplay, a 7,68/10 que se obtuvo después de trabajar con educaplay. Por lo tanto, esta herramienta multimedia si favorece el logro de aprendizajes de química general.

Resultados de la guía de observación aplicada a los estudiantes del segundo semestre de la carrera de biología, química y laboratorio sobre el desarrollo de competencias específicas de las asignaturas de química y física general desarrolladas usando la metodología tradicional y herramientas multimedia.

\section{Tabla 2}

Describe la estructura de la tabla periódica y relaciona la posición de los elementos con sus propiedades y configuración electrónica respectiva.

\begin{tabular}{ccrccc}
\hline \multicolumn{3}{c}{ Metodología tradicional } & \multicolumn{3}{c}{ Uso de Educaplay } \\
\hline Siempre & rara vez & nunca & siempre & rara vez & nunca \\
\hline 4 & 6 & 12 & 12 & 8 & 2 \\
\hline
\end{tabular}

Fuente: Ficha de observación aplicada a de los estudiantes de segundo semestre 
El uso de Educaplay como recurso didáctico permitió que un mayor número de estudiantes logren relacionar la posición de los elementos en la tabla periódica y por tanto la estructura de la misma, esto se debió en su mayoría a la realización de actividades didácticas como: ruleta de letras, relacionar columnas lo cual favoreció el aprendizaje de química, como lo señala María Montesori las actividades planteadas no es un simple pasatiempo, ni una sencilla fuente de información, es más que eso, es material didáctico para enseñar. Las actividades diseñadas estuvieron ideadas para captar la curiosidad del educando.

\section{Tabla 3}

Explica e interpreta los modelos atómicos

\begin{tabular}{crrrrrr}
\hline \multicolumn{3}{c}{ Metodología tradicional } & \multicolumn{3}{c}{ Uso de Educaplay } \\
\hline Siempre & rara vez & nunca & siempre & rara vez & nunca \\
\hline $\mathbf{8}$ & 9 & 5 & 14 & 6 & 2
\end{tabular}

Fuente: Ficha de observación aplicada a de los estudiantes de segundo semestre

Esta temática por lo general se aborda a partir de la realización de modelos atómicos con material concreto, sin embargo, los resultados obtenidos con el uso de educaplay, principalmente a través del videoquiz, fueron muy alentadores, los resultados obtenidos se relacionan con lo manifestado por Lev Vygotsky la importancia que tiene los procesos de innovación ha llevado frecuentemente a asociar relación de recursos con innovación educativa.

\section{Tabla 4}

Establece la relación entre las fuerzas intermoleculares y los estados de agregación que presenta la materia.

\begin{tabular}{cccccc}
\hline \multicolumn{3}{c}{ Metodología tradicional } & \multicolumn{3}{c}{ Uso de Educaplay } \\
\hline Siempre & rara vez & nunca & siempre & rara vez & nunca \\
\hline 4 & 14 & 4 & 13 & 7 & 2 \\
\hline
\end{tabular}

Fuente: Ficha de observación aplicada a de los estudiantes de segundo semestre

Los resultados del uso de las TICs en el aula de clase son superiores a los obtenidos por la metodología tradicional, lo cual va en relación con lo mencionado Hernández 2014, donde menciona que el uso de la TICs facilita la comprensión de conceptos (Hernández y Rodríguez, 2014, p.39), porque el alumno a través de la repetición, asociación y observación puede retroalimentar, relacionar conceptos, y propiciar de ésta forma un mejor aprendizaje. 


\section{Tabla 5}

Enuncia los principios que describen los distintos tipos de enlace químico.

\begin{tabular}{cccccc}
\hline \multicolumn{3}{c}{ Metodología tradicional } & \multicolumn{3}{c}{ Uso de Educaplay } \\
\hline Siempre & rara vez & nunca & siempre & rara vez & nunca \\
\hline 3 & 13 & 6 & 15 & 5 & 2
\end{tabular}

Fuente: Ficha de observación aplicada a de los estudiantes de segundo semestre

El uso de actividades interactivas diseñadas en educaplay permitió que los alumnos logren entender de mejor manera la formación de enlaces y los procesos de donación o coparticipación de electrones, el uso de imágenes en movimiento, donde se puede determinar el movimiento de los electrones de un átomo a otro facilitan la comprensión de estos procesos.

\section{Tabla 6}

Interpreta los fenómenos físicos que ocurren en el ambiente.

\begin{tabular}{cccccc}
\hline \multicolumn{3}{c}{ Metodología tradicional } & \multicolumn{3}{c}{ Uso de Educaplay } \\
\hline Siempre & rara vez & nunca & siempre & rara vez & nunca \\
\hline 3 & 11 & 6 & 12 & 5 & 3
\end{tabular}

Fuente: Ficha de observación aplicada a de los estudiantes de segundo semestre

EDUCAPLAY permitió establecer criterios y compartir experiencias entre los estudiantes a través del icono de dialogo lo cual ha permitido saber de fenómenos que no todos lo hemos visto, la metodología tradicional no ayuda en la socialización oportuna y con evidencia de tales fenómenos.

\section{Tabla 7}

Diferencia los sistemas de unidades utilizados en el estudio de la física

\begin{tabular}{cccccc}
\hline \multicolumn{3}{c}{ Metodología tradicional } & \multicolumn{3}{c}{ Uso de Educaplay } \\
\hline Siempre & rara vez & nunca & siempre & rara vez & nunca \\
\hline $\mathbf{2}$ & 10 & 8 & 12 & 9 & 1
\end{tabular}

Fuente: Ficha de observación aplicada a de los estudiantes de segundo semestre

Por medio del desarrollo de actividades con educaplay se obtuvo resultados eficientes en los procesos de diferenciación de los sistemas de unidades utilizados en el estudio de la física en comparación con la metodología tradicional, debido a que con la conformación de esquemas conceptuales los estudiantes logran profundizar y solidificar sus conocimientos con capacidad de análisis y síntesis, lo cual va en relación con lo establecido por Novak quien establece que los 
mapas conceptuales son un medio adecuado para que el alumnos pueda visualizar conceptos y establecer relaciones jerárquicas entre conceptos. (Novak, 1998, pg. 11).

\section{Tabla 8}

Discrimina el instrumento de medida apropiado para cada magnitud.

\begin{tabular}{cccccc}
\hline \multicolumn{3}{c}{ Metodología tradicional } & \multicolumn{3}{c}{ Uso de Educaplay } \\
\hline Siempre & rara vez & nunca & siempre & rara vez & nunca \\
\hline $\mathbf{2}$ & 12 & 6 & 12 & 6 & 2
\end{tabular}

Fuente: Ficha de observación aplicada a de los estudiantes de segundo semestre

El uso de la herramienta educativa Educaplay permitió identificar los instrumentos de medida que se utilizan para las diferentes magnitudes y lograr ubicarlos y recordarlos, para lo cual la actividad de completar produjo mejores resultados; la metodología tradicional no da apertura para este tipo de actividades lúdica.

Aprender a Aprender de forma divertida a través de la herramienta Didáctica EDUCAPLAY permite al estudiante sentirse distendido, motivado, abierto al proceso pedagógico, que contribuirá a un aprendizaje significativo y a formar personas útiles a la sociedad.

\section{Conclusiones}

El uso de la herramienta multimedia educaplay contribuyó a mejorar el aprendizaje de Química General en los estudiantes del segundo semestre de la Carrera de Biología, Química y Laboratorio de la Facultad de Ciencias de la Educación Humanas y Tecnologías de la Universidad Nacional de Chimborazo; siendo de suma importancia para los actores del aprendizaje.

Al analizar la herramienta multimedia Educaplay se establece que es apta para el aprendizaje de Física General, debido a su dinamismo, didáctica y motivación que aporta, además a sacar de la monotonía a estudiantes y docentes con la manipulación de nuevas técnicas.

El uso de actividades lúdicas como crucigrama, videoquiz, ruleta de palabras, entre otras, estimulan el aprendizaje de Química y Física General debido a su versatilidad y fácil manejo, además contribuye a aumentar la motivación el aula.

El uso de Educaplay como recurso didáctico para el aprendizaje de Química General, permitió desarrollar en los estudiantes procesos mentales superiores a través de la manipulación de actividades afines con la materia. 
A través de la utilización de la herramienta didáctica Educaplay se mejora la motivación del alumno hacia la asignatura de Física, lo cual se reflejó en un mejor aprendizaje, desarrollando al mismo tiempo la creatividad y reflexión en el análisis de los fenómenos físicos.

\section{Referencias Bibliográficas}

Domingo, M., \& Perez, M. (2011). Aulas 2.0 y uso de las TIC en la práctica docente. Red de Revistas Científicas de América Latina y el Caribe, España y Portugal, pp. 169-175.

Gómez, D. (2012). Incorporación de las TICs al aula de química. Studiositas, pp. 22-28.

Hernández, M., Rodríguez, V., \& Francisco, P. (2014). las tecnologías de la información y la comunicación (tics) en la enseñanza-aprendizaje de la química orgánica a través de imágenes, juegos y video. Formación universitaria, pp. 31-40.

Novak, J. (1998). Aprendiendo a aprender. Barcelona: Martínez Roca.

Pérez, N. (2014). Desarrollo de las capacidades de comprensión y producción de textos en el área de inglés en alumnos de 1er. Año de secundaria de una institución educativa particular de lima. Tesis de postgrado. Pontificia Universidad Católica Del Perú. Lima

Riascos, S., \& Quintero, M. ä. (2009). Las TIC en el aula: percepciones. Educación y Educadores, pp. 153-157.

Riveros, V., \& Mendoza, M. I. (2005). Bases teóricas para el uso de las TIC. Encuentro Educacional, pp. 315-336.

Rodríguez, M., \& García, E. (2007). Estrategias de aprendizaje y sus particularidades de en lenguas extranjeras. Cuba.

Sepúlveda, L. (2014). La incorporación de la tecnología en la enseñanza de la química. Tesis de pregrado. Universidad del Valle. Cali, Colombia. 